\title{
Entropy-Driven Formation of a Chiral Liquid-Crystalline Phase of Helical Filaments
}

\author{
Edward Barry, Zach Hensel, and Zvonimir Dogic \\ Rowland Institute at Harvard, Harvard University, Cambridge, Massachusetts 02142, USA \\ Michael Shribak and Rudolf Oldenbourg \\ Marine Biological Laboratory, Woods Hole, Massachusetts 02543, USA
}

(Received 2 September 2005; published 11 January 2006)

\begin{abstract}
We study the liquid-crystalline phase behavior of a concentrated suspension of helical flagella isolated from Salmonella typhimurium. Flagella are prepared with different polymorphic states, some of which have a pronounced helical character while others assume a rodlike shape. We show that the static phase behavior and dynamics of chiral helices are very different when compared to simpler achiral hard rods. With increasing concentration, helical flagella undergo an entropy-driven first order phase transition to a liquid-crystalline state having a novel chiral symmetry.
\end{abstract}

PACS numbers: $82.70 .-\mathrm{y}, 61.30 . \mathrm{Vx}$

Molecules with chiral symmetry cannot be superimposed on their mirror image. Such molecules can assemble into a variety of complex chiral structures of importance to both physics and biology [1]. Chirality has a special prominence in the field of liquid crystals, and the presence of a chiral center can dramatically alter the liquid-crystalline phase behavior and its material properties [2]. For example, achiral rods form a nematic phase with long-range orientational order. However, rearranging a few atoms to create a microscopically chiral molecule can transform a nematic into a cholesteric phase. Locally, a cholesteric phase has a structure in which molecules are organized in layers. Within a layer, the molecules are parallel to each other, while the molecular orientation is slightly rotated between two adjacent layers. This order at least partially satisfies the pair interaction between neighboring chiral molecules which tends to twist their mutual orientation. Even for the fairly simple example of a cholesteric phase, it is difficult to establish a rigorous relation between the microscopic chirality of the constituent molecules and the macroscopic chirality characterized by the cholesteric pitch $[3,4]$.

In stark contrast to our poor understanding of the cholesteric phase, microscopic theories of nematic liquid crystals have been very fruitful [5]. Onsager realized that a simple fluid of concentrated hard rods will form a stable nematic phase. Using his theory, it is possible to predict the macroscopic phase behavior of a nematic suspension of hard rods from microscopic parameters such as rod concentration and rod aspect ratio. Because of the dominance of repulsive interactions, phase transitions within the Onsager model belong to a class of entropy-driven phase transitions. Inspired by the success of the Onsager theory, Straley made the first attempt at formulating a microscopic theory of the cholesteric phase [4]. In this work, hard-rod interactions are extended to threaded rods, which have an appearance similar to screws. The excluded volume between two threaded rods is at a minimum not when they are parallel to each other but when they approach each other at an angle at which their chiral grooves can interpenetrate. This results in the formation of a cholesteric phase that is, as in the Onsager model, entirely driven by entropic excluded volume interactions.

Biopolymers such as DNA, actin, tobacco mosaic virus (TMV), and $f d$ are good experimental systems to study certain aspects of liquid-crystalline ordering of colloidal rods [6-8]. In particular, monodisperse rodlike viruses were used to quantitatively test the Onsager theory [9]. However, the usefulness of these systems in elucidating the microscopic origin of chirality is severely limited due to the inability to precisely control their microscopic chiral structure. While almost all biopolymers have chiral stucture, some of them form a nematic phase ( $p f 1$ and TMV), while others form a cholesteric phase $[f d$, poly(ehtylene glycol)- $f d$, and DNA] [6,7]. The reason for this behavior remains a mystery.

In this Letter, we use flagella isolated from prokaryotic bacteria to experimentally study the phase diagram of a concentrated suspension of helices. Flagellar filaments are macromolecular structures assembled from a single protein called flagellin. A unique advantage of a bacterial flagellum is that its helical shape is precisely regulated and depends on the flagellin amino acid sequence and the $p \mathrm{H}$ and temperature of the suspension (Fig. 1) [10,11]. Moreover, it can be tuned in a number of discrete steps from essentially achiral hard rods to highly twisted helices. Using this system, we can readily control the microscopic chirality of the flagella and relate this to its liquidcrystalline phase behavior. Unlike the predictions of the Straley model, we find that the liquid-crystalline behavior of helices is dramatically different from that of straight rods. They form a liquid-crystalline phase with a novel chiral symmetry which we call a conical phase. The symmetry of this phase is similar to a structure that can occur when a cholesteric phase is placed in a magnetic field along its twist direction and the molecules consequently tip along 


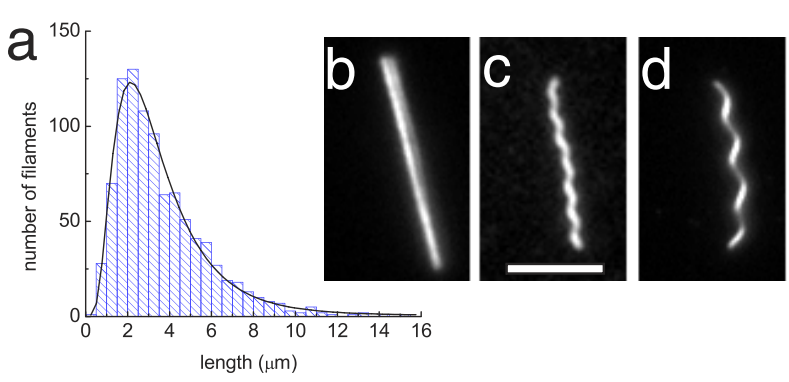

FIG. 1 (color online). (a) Length distribution of flagella isolated from strain SJW1103. (b) Fluorescently labeled straight flagella isolated from strain SJW1665. (c) Mutant flagella SJW2869 characterized by its pitch $(P=1.1 \mu \mathrm{m})$ and diameter $(D=0.160 \mu \mathrm{m})$. The average contour length is $4.01 \pm 2.5 \mu \mathrm{m}$. (d) Wild-type flagella SJW1103 $(P=2.4 \mu \mathrm{m}, D=0.4 \mu \mathrm{m})$. The average length is $3.6 \pm 2.2 \mu \mathrm{m}$. The scale bar indicates $5 \mu \mathrm{m}$.

the applied field [12]. Experimentally, this case is rarely observed, since Frank elastic constants have to satisfy the condition $K_{22}>K_{33}$. We also show that the dynamics of helices in a conical phase is very different from the dynamics of achiral rods in a nematic. Previous studies of ordering in flagella were limited to achiral rods for the purpose of structure determination [13].

The phase diagram of flagella suspensions is highly dependent on their length distribution, which in turn is sensitive to purification methods. Bacteria grown to a log-phase are sedimented at $8000 \mathrm{~g}$ and redispersed by repeated pipetting with $1 \mathrm{~mL}$ pipette. A foamy solution is vortexed at the highest power setting for 5 min (Genie 2 Vortex) and centrifuged at $8000 \mathrm{~g}$ for $20 \mathrm{~min}$. The supernatant contains flagellar filaments, which are purified and concentrated by two centrifugation steps at $100000 \mathrm{~g}$ for $1 \mathrm{~h}$. Filaments are labeled with a fluorescent dye (TAMRASE, Molecular Probes). To obtain the histogram of filament lengths, we dry a suspension of flagella in $1 \%$ methylcellulose onto a coverslip which flattens the 3D helices. Unless otherwise noted, all the images were taken with a Nikon polarization microscope E600 equipped with fluorescence components.

At concentrations below $17 \mathrm{mg} / \mathrm{ml}$, wild-type flagella SJW1103 form isotropic suspensions. Above this concentration, samples initially appear in a uniformly birefringent metastable state. Over a period of days, bright birefringent droplets (tactoids) start phase separating from a dark isotropic background [Fig. 2(a)]. These droplets exhibit a well defined striped pattern with a $2.4 \mu \mathrm{m}$ periodicity. With increasing flagella concentration, the volume fraction of tactoids increases, and at concentrations above $29 \mathrm{mg} / \mathrm{ml}$ the samples appear birefringent everywhere with no hint of coexisting isotropic solution. These single phase samples have a polydomain texture with a very small domain size. Moreover, the domains do not anneal over time. The best way to obtain macroscopically aligned samples [Fig. 2(b)] is to prepare a coexisting sample and let it bulk phase
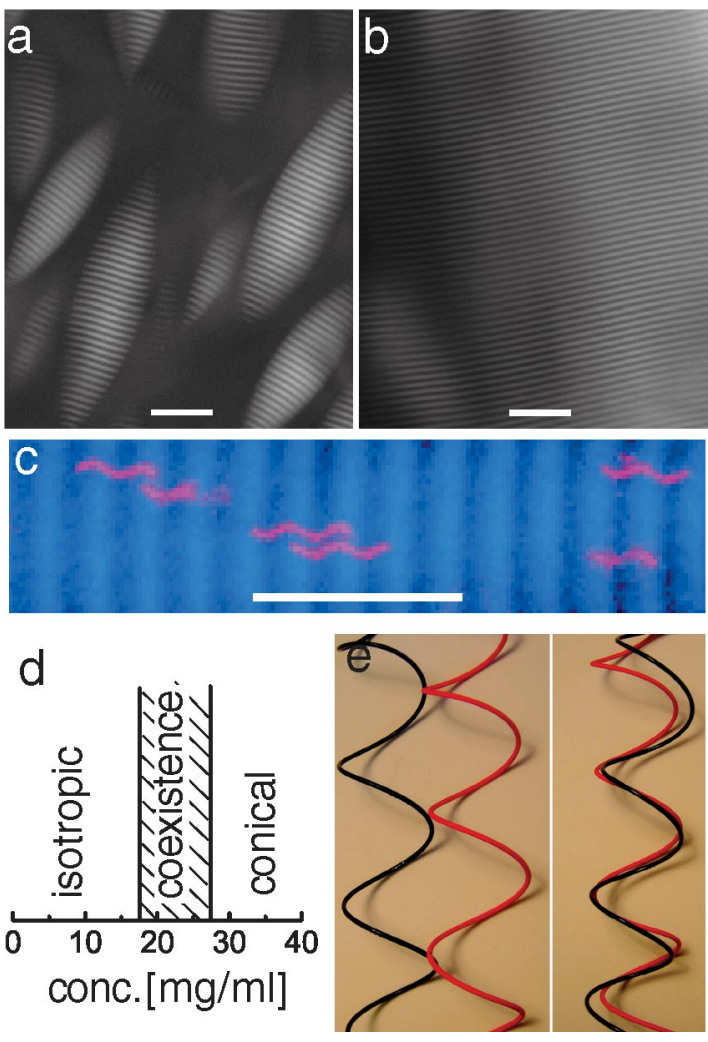

FIG. 2 (color). (a) Coexistence between isotropic and conical phase in flagella SJW1103 at $20 \mathrm{mg} / \mathrm{ml}$ imaged with a polarization microscope. The scale bar is $20 \mu \mathrm{m}$. (b) A single phase imaged with a polarization microscope; the scale bar is $20 \mu \mathrm{m}$. (c) Fluorescently labeled flagella dissolved in a conical phase of unlabeled flagella. The fluorescent image was overlaid above a polarization microscope image. The scale bar is $10 \mu \mathrm{m}$. (d) Tentative phase diagram of flagella SJW1103. (e) Excluded volume between two helical rods out of phase and in phase with respect to each other.

separate over a period of a few weeks. From these observations, we obtain a tentative phase diagram of flagella suspensions, shown in Fig. 2(d). The slow dynamics and possible presence of a nonequilibrium glassy phase complicate the accurate determination of the upper binodal point. The large width of the isotropic-liquid-crystalline coexistence is a consequence of the polydispersity of the filaments [14]. Over a temperature range from 0 to $40^{\circ} \mathrm{C}$, the phase diagram does not change, indicating that temperature independent repulsive interactions dominate the phase behavior. The well defined shape of the tactoids indicates that these are equilibrium structures and are not formed due to irreversible aggregation. Tactoids such as these are frequently observed in coexisting samples of a wide variety of rodlike colloids [15].

The most surprising feature of the liquid-crystalline phase of flagella SJW1103 is a well defined onedimensional periodicity with a wavelength that corresponds to the pitch of its constituent flagella. The striped pattern is observed only with polarization microscopy 
which is sensitive to the local orientation of the molecules. When viewed with differential interference contrast microscopy, which is sensitive to concentration differences, no spatial variations are observed. Therefore, there are only orientational variations and no density variations along the long axis of the tactoid. In addition, fluorescent images reveal that the filaments are always in phase with respect to each other [Fig. 2(c)]. These observations lead us to propose a liquid-crystalline structure in which the helical filaments are intercalated with each other and the liquidcrystalline director follows the path set by the geometry of constituent molecules. While there are no 1D density variations associated with this order, there are clear onedimensional orientational variations which make the director span the surface of a cone. Therefore, we call this liquid-crystalline phase a conical phase, which was first described in Ref. [12]. Polydispersity of the helices suppresses the formation of a positional smectic. We emphasize that this organization of molecules is very different from cholesterics, where the director twists perpendicularly to its local direction. It is intuitively clear how excluded volume interactions result in the formation of a conical phase. The excluded volume between two helices is much larger when they are out of phase than when they are in phase with each other [Fig. 2(e)]. This is the driving force for the formation of the conical phase.

Figure 3 illustrates the behavior of the liquid-crystalline phase as the geometry of the filaments changes. As expected, straight filaments form a nematic [Fig. 3(a)]. Slightly helical filaments (strain SJW2869) form a conical phase with spacing of $1.1 \mu \mathrm{m}$, corresponding to the pitch of an isolated flagellum. For this sample as well as for flagella SJW1103, we observe a direct transition from isotropic to conical phase with no intermediate nematic phase. It is also possible to envision a scenario in which helical rods first form a nematic (cholesteric) phase and only upon further compression, when helices begin to
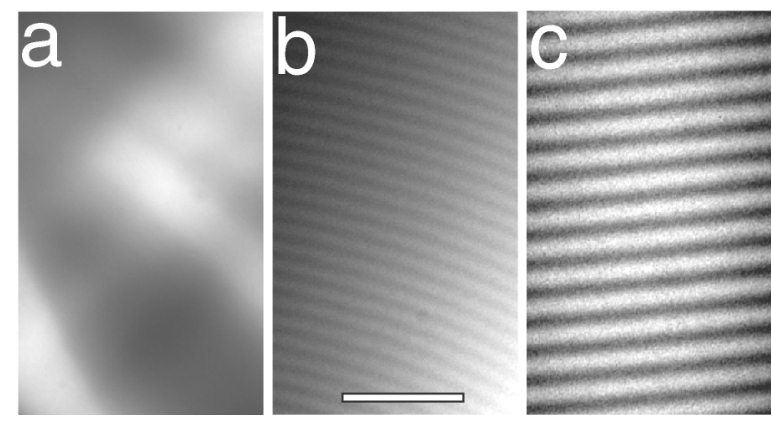

FIG. 3. Polarized light images of liquid-crystalline flagella suspensions. (a) Nematic liquid crystal phase observed in straight flagella SJW1665. (b),(c) Conical phase formed from helical flagella SJW2869 and SJW1103, respectively. Bright and dark stripes indicate differing director orientations. The images in (b) and (c) exhibit one-dimensional periodicities of 1.1 and $2.4 \mu \mathrm{m}$, respectively. The scale bar is $10 \mu \mathrm{m}$. intercalate, undergo a second transition to a conical phase. The flagella SJW2869 exhibit an isotropic phase below $7 \mathrm{mg} / \mathrm{ml}$, coexisting samples between 7 and $26 \mathrm{mg} / \mathrm{ml}$, and a conical phase above $26 \mathrm{mg} / \mathrm{ml}$. The liquidcrystalline phase in SJW2869 occurs at much lower concentrations when compared to SJW1103 despite similar length distribution. This might be due to the larger ratio of contour length over diameter of flagella SJW2869.

To gain a better understanding of the conical phase, we have analyzed our samples with quantitative 2D polarization microscopy (LC-PolScope), which simultaneously provides detailed spatial maps of the retardance and orientation of the slow axis of birefringence [16]. Similar to traditional polarized light images, the retardance and orientation maps show striped patterns, indicating the systematic variations in director orientation. In some cases, the striped pattern visible in the retardance image [Fig. 4(b)] exhibits half the period (or twice the spatial frequency) compared to the orientation image [Fig. 4(a)]. This is consistent with the liquid-crystalline director following a helical trajectory in the image plane. By way of explanation, the measured retardance in an LC-PolScope image is modulated by the inclination of the director with respect to

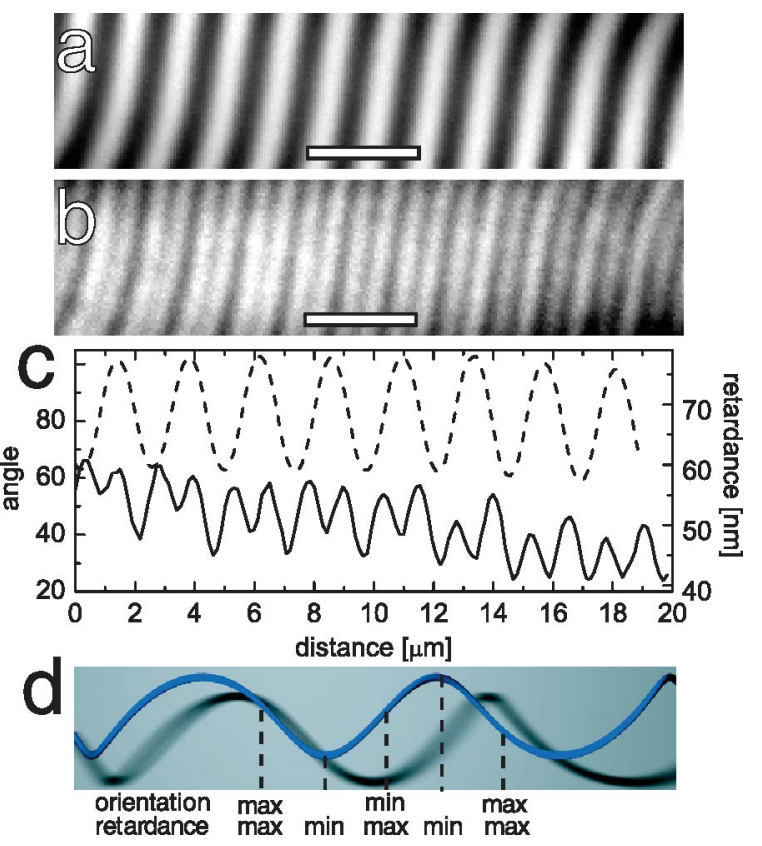

FIG. 4 (color). Analysis of a conical phase of flagella SJW1103 based on LC-PolScope images. (a),(b) The images show a map of the orientation of the slow axis of the birefringence and a retardance map, respectively. The scale bars are $5 \mu \mathrm{m}$. (c) Line profile along the striped pattern of the orientation image (dashed line) and retardance image (solid line). When comparing the retardance to the orientation image, frequency doubling is observed. (d) Dashed lines represent the location along a helical director where the minimum and maximum of the orientation and retardance occurs. From this schematic, it is clear that retardance has twice the periodicity of orientation. 


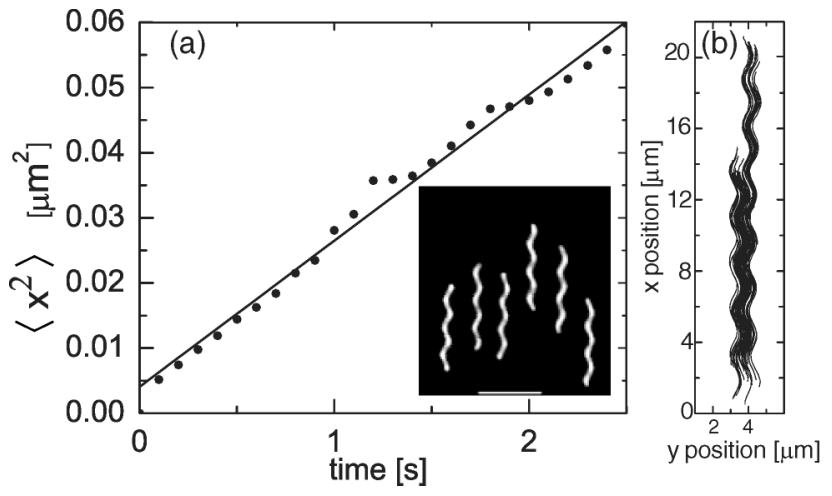

FIG. 5. Dynamics of a fluorescently labeled flagellum in a conical phase. The flagellum exhibits effective translation motion through rotation similar to the behavior of a nut on a bolt. (a) Mean square displacement of the phase angle of an individual flagellum. Inset: A time sequence of a diffusing flagellum taken over a period of several minutes. (b) Overlapped trajectories reconstituted of a flagellum diffusing in a conical phase.

the object plane that is in focus. When the director is inclined to the object plane, light travels more parallel to the director, and the measured retardance decreases compared to when the director is parallel to the object plane. When the director follows a helical path, with the helix axis parallel to the object plane, the director attains a parallel orientation twice within one helical pitch and attains positive and negative inclination angles in between. Because the reduction is the same for positive and negative inclination angles, the observed periodicity in the retardance image is half the helical pitch. If the helix axis is not parallel to the object plane, the magnitudes of the extreme positive and negative inclination angles are unequal and the retardance minima are unequal, as observed in Figs. 4(b) and 4(c). It is interesting to note that the appearance of twice the fundamental frequency is usually not apparent in images recorded using a traditional polarization microscope (Figs. 2 and 3). The LC-PolScope generates independent retardance and orientation maps and makes the doubling of the frequency in retardance images visible.

In nematics, the broken orientational symmetry results in anisotropic diffusion coefficients with rods preferentially diffusing along the nematic director [17]. In a similar fashion, the helical symmetry of the conical phase results in an unusual diffusion of constituent helices. Figure 5 shows a time sequence and reconstructed trajectories of an isolated flagellum diffusing in a conical phase. As shown in Fig. 5(b), all the peaks and valleys are in register, indicating that translational diffusion is strictly coupled to rotational diffusion. This is similar to the movement of a "nut on a bolt." For diffusion along the helix axis, the mean square displacement of the filament end points increase linearly with time, leading to a translational diffusion constant of $0.023 \mu \mathrm{m}^{2} / \mathrm{sec}$. For a translational displacement that corresponds to one helical pitch, the filament rotates by $2 \pi$. Hence, the translational diffusion of $0.023 \mu \mathrm{m}^{2} / \mathrm{sec}$ corresponds to a rotational diffusion of $0.16 \mathrm{rad}^{2} / \mathrm{sec}$. We find that the diffusion depends on the length of the filaments with smaller filaments diffusing faster than longer ones. In addition to diffusion along the helical axis, we observe significant lateral diffusion, which excludes the presence of columnarlike long-range order.

In conclusion, we note that the behavior of hard rods, the simplest model system of liquid crystals, has been studied extensively for several decades. In the present Letter, we use well regulated helical filaments to demonstrate that the static phase behavior and dynamics of a concentrated suspension of helical filaments are remarkably different from those of a suspension of hard rods. Our work addresses the fundamental question of the molecular origins of the varied liquid-crystalline mesophases that are found in biological systems and are believed to play an important role in their function.

We thank Eric Grelet, Linda Stern, Tom Lubensky, Seth Fraden, and Randy Kamien for useful comments and Noreen Francis for bacterial strains. M. S. and R. O. are supported by NIH Grant No. EB002583.

[1] A. B. Harris et al., Rev. Mod. Phys. 71, 1745 (1999); R. L. B. Selinger et al., Phys. Rev. Lett. 93, 158103 (2004); F. Livolant and A. Leforestier, Biophys. J. 78, 2716 (2000).

[2] R. B. Meyer et al., J. Phys. Lett. 36, L69 (1975); S. R. Renn and T. C. Lubensky, Phys. Rev. A 38, 2132 (1988); R. D. Kamien and D. R. Nelson, Phys. Rev. Lett. 74, 2499 (1995); E. Grelet et al., Phys. Rev. Lett. 86, 3791 (2001).

[3] A. B. Harris et al., Phys. Rev. Lett. 78, 1476 (1997).

[4] J. P. Straley, Phys. Rev. A 14, 1835 (1976).

[5] L. Onsager, Ann. N.Y. Acad. Sci. 51, 627 (1949).

[6] Z. Dogic and S. Fraden, Langmuir 16, 7820 (2000); E. Grelet and S. Fraden, Phys. Rev. Lett. 90, 198302 (2003).

[7] F. Livolant, Physica (Amsterdam) 176A, 117 (1991).

[8] R. Podgornik et al., Proc. Natl. Acad. Sci. U.S.A. 93, 4261 (1996).

[9] R. Oldenbourg et al., Phys. Rev. Lett. 61, 1851 (1988).

[10] R. Kamiya et al., Symp. Soc. Exp. Biol. 35, 52 (1982); S. V. Srigiriraju and T. R. Powers, Phys. Rev. Lett. 94, 248101 (2005).

[11] S. Kanto et al., J. Mol. Biol. 219, 471 (1991).

[12] R. B. Meyer, Appl. Phys. Lett. 12, 281 (1968); R. D. Kamien, J. Phys. II 6, 461 (1996).

[13] I. Yamashita et al., J. Mol. Biol. 278, 609 (1998).

[14] H. H. Wensink and G. J. Vroege, J. Chem. Phys. 119, 6868 (2003).

[15] P. Prinsen and P. van der Schoot, Phys. Rev. E 68, 021701 (2003).

[16] R. Oldenbourg and G. Mei, J. Microsc. 180, 140 (1995); M. Shribak and R. Oldenbourg, Appl. Opt. 42, 3009 (2003).

[17] M. P. Lettinga et al., Europhys. Lett. 71, 692 (2005). 